\title{
Hypothyroid Patients with Positive Thyroid Antibodies and Relation to Nerve Conduction Study Findings
}

\author{
Ila Venkata Padma ${ }^{1}$, Sanghamitra Panda ${ }^{2}$ \\ ${ }^{1}$ Assistant Professor Physiology Shadan Institute of Medical Sciences Hyderabad India, ${ }^{2}$ Professor HOD \\ Department of Physiology Shadan Institute of Medical Sciences Hyderabad India
}

\begin{abstract}
Neuropathies are common manifestations of more than half the patients attending medical out patient departments. These neuropathies could be due to many causes. Some of the patients are not diabetics or alcoholics and thyroid profile revealed hypothyroidism. Most of the patients were not on thyroid supplementation and some are on irregular treatment. Primary symptoms are vague not pointing to either thyroid deficiency or diabetes mellitus complained of lethargy, pain in the fingers stabbing in nature, wight gain, dryskin ,constipation . Nerve conduction studies on median and ulnar nerves in these patients without diabetes and other deficiency disorders point to miss hypothyroid state in these patients, as stress is put on diagnosing more common conditions like diabetes mellitus. If these conditions are excluded it is not difficult to diagnose hypothyroid conditions.
\end{abstract}

Key words : Anti-thyroid antibodies, nerve conduction studies, TPO, thyroid peroxidise antibodies, thyroidautoimmunity.

\section{Introduction}

Hashimoto's thyroiditis (HT), the most frequent autoimmune thyroid disorders (AITDs), is the leading cause of hypothyroidism in the iodine-sufficient areas of the world. About $20-30 \%$ of patients suffer from HT, whose cause is thought to be a combination of genetic susceptibility and environmental factors that causes the loss of immunological tolerance, with a consequent autoimmune attack to the thyroid tissue and appearance of the disease.

The pathologic features of lymphocytic infiltration, especially of $\mathrm{T}$ cells, and follicular destruction are the histological hallmark of autoimmune thyroiditis (AIT), that lead to gradual atrophy and fibrosis. An important role in the immune-pathogenesis of AITDs is due to chemokines and cytokines.

\section{Corresponding author: \\ Dr Ila Venkata Padma}

Asst Professor Physiology

Shadan Institute of Medical Sciences

Hyderabad, Telangana, India
Inflammatory cytokines and in particular IL6 and $\mathrm{TNF} \alpha$, released during local or systemic inflammation are the main molecular candidates in driving $\mathrm{TH}$ (thyroid hormone) tissue alterations ${ }^{[1]}$. Thus tissue concentrations of THs and their molecular mediators possibly more effectively reflects the functional state of the thyroid system (TS) at target than circulating hormones ${ }^{[2]}$.

Major concepts of Hashimoto's neuropathy are vasculitis theory, hormone dysregulation theory explaining the disease via direct action of the autoantibodies against various thyroid (thyroperoxidase, thyroglobulin, and TSH-receptor) and several extrathyroid antigens (alpha-enolase and other enzymes, gangliosides and MOG-protein, onconeuronal antigens) all of them expressed in the brain ${ }^{[3]}$.

A thyroid antibodies test is used to help diagnose autoimmune disorder of the thyroid. Thyroid peroxidise antibodies (TPO), Thyroglobulin antibodies (Tg) , presence is a sign of Hashimoto disease.

Auto antibodies (proteins of the immune system that aberrantly react against the body's own cells) that bind to fibroblasts to produce and release chemical 
signals and biologic materials that lead to swelling and congestion of the tissues affected. These auto antibodies can be measured in the blood to help monitor disease activity and severity does not always correlate with thyroid hormone levels ${ }^{[4]}$.

Thyroid peroxidise antibodies should be measured if the TSH is borderline high on two occasions and there are no overt symptoms of hypothyroidism as this will guide the frequency of future monitoring ${ }^{[5]}$. This test is commonly used to confirm or exclude Hasimoto's thyroiditis as the reason for hypothyroidism.

Thyroid peroxidase is the major antigen in human Hashimoto's disease, and anti-TPO antibodies induce complement-dependent cytotoxicity. Furthermore, antibodies against complement (anti-C1q) are detected in patients with Hashimoto's disease. These antibodies can damage the thyroid follicular cells directly by activating the complement cascade.They are correlated with thyroid-stimulating hormone (TSH) levels ${ }^{[6]}$. Modelling of thyroid peroxidase reveals insights into its enzyme function and autoantigenicity ${ }^{[7]}$.

TPO is oxidized by $\mathrm{H}_{2} \mathrm{O}_{2}$ and then only TPO can oxidize iodide ions. Oxidized iodide ions bind to tyrosyl residues of thyroglobulin (TG). Formation of T4 and T3 iodothyronines is an effect of oxidation and coupling of hormonogenic iodotyrosines.

As the TPO enzyme is a heme peroxidase, it cannot oxidize the substrate without having been oxidized. To oxidize TPO, the $\mathrm{H}_{2} \mathrm{O}_{2}$ molecule is necessary. The $\mathrm{H}_{2} \mathrm{O}_{2}$ molecule is generated only at the apical surface of thyrocytes, and the TPO molecules that are present at this surface are activated.

Intracellular Content and TH Genomic Effects In the brain , T4 is taken up by astrocytes, probably by OATP1C1 (organic anion transporter $1 \mathrm{C} 1$ ) transporter.

Intracellularly the enzyme type-2 iodothyronine deiodinase (D2) generates T3 by catalyzing the removal of ring iodine atoms in the cytosol, from which T3 easily reaches the nucleus. In other cell types, such as neurons, D3 catalyzes the removal of the outer- or inner-ring iodine atoms to produce T2 or the inactive form rT3, respectively ${ }^{[8]}$. T3-target cells also include neurons, oligodendrocytes, and many other cell types in the nervous tissue.

This is linked to the extremely complex metabolism of THs in the CNS, and particularly to the interplay between $\mathrm{TH}$ membrane transporters and deiodinase activity. The THs actually cross the BBB partially via gaps among the cells that compose the blood-brain barrier, but mainly using transporters, thus through a saturable process ${ }^{[9]}$. Thus, tissue hypothyroidism during inflammation could impact on cellular processes, requiring an appropriate $\mathrm{TH}$ drive, including $\mathrm{OPC}$ differentiation into myelinating $\mathrm{OL}^{[10]}$. These results and considerations form the basis of preclinical studies attempting to correct myelination and remyelination failure in experimental conditions characterized by intense tissue inflammation and TS signaling dysregulation, starting from the fact that the block of TH-dependent OPC differentiation has been described in both perinatal and adult animal and human samples ${ }^{[1]]}$.

Oligodendrocyte progenitor cell (OPC) also responsible for myelin repair during adulthood as remyelination is the only true regenerative capability of the $\mathrm{CNS}^{[12]}$. OPCs are present in the mature CNS, where they represent a major proliferating cell population, constituting $5 \%$ of the total cell population ${ }^{[13]}$. In response to myelin loss or increased demand, "adult" OPCs have the capacity to differentiate into mature myelinating OL ${ }^{[14]}$ to guarantee an appropriate white matter turnover. This significant capability, demonstrated in physiological conditions, led to the so-called recapitulation hypothesis for myelin repair which suggested that remyelination after myelin damage follows several myelination steps and molecular mechanisms occurring during developmental myelination ${ }^{[15]}$. Several studies have confirmed the impact of $\mathrm{TH}$ on myelin development, homeostasis, and repair focusing on the regulation of $\mathrm{TH}$ tissue signaling under physiological and pathological conditions affecting myelination and/or myelin repair during early postnatal age and during adulthood.

"Tissue hypothyroidism in the CNS" ${ }^{[16]}$ In a recent paper, it was suggested a direct link between cytokines, D3 expression, and OPC differentiation failure ${ }^{[17]}$.

Experiments have demonstrated that under cytokine exposure a dramatic rise in the number of D3positive cells was observed in OPC derived from neural 
precursors. Using the deiodinase blocker iopanocic acid (IOP) in order to decrease D3 activity and then increase T3 intracellular availability. This treatment was able to restore the capability of the OPC culture to express differentiation markers (CNPase and MBP), also restoring OL mature morphology. It may thus be concluded that IOP inhibits D3, thereby restoring an appropriate $\mathrm{T} 3$ content in OPCs that overcome the cytokine-induced OPC differentiation block. This effect could explain the positive results obtained by in vivo TH supplementation in experimental models of inflammatory demyelination. Indeed, these studies indicated that $\mathrm{TH}$ supplementation restores the expression on a number of TH-dependent genes which are key determinants for OPC maturation the activation of which is necessary to promote remyelination $^{[18]}$.

The remyelination failure in neurological conditions characterized by intense inflammation is likely due not only to a loss of OL, but also to OPC differentiation block. The intense inflammation also causes a complex dysregulation of TH signaling in the CNS, characterized by D3 upregulation and TR downregulation, possibly leading to tissue hypothyroidism. Since T3 is a critical player for OPC differentiation, the decreased availability of the active $\mathrm{T} 3$ in the tissue, particularly in OPC during inflammation, might contribute to OPC differentiation failure. Thus, the possibility of including $\mathrm{TH}$ as adjuvant therapy in diseases and injuries during the acute inflammatory phase might well be considered. It was demonstrated that pulsed $\mathrm{TH}$ treatment increases the MBP content, restores myelin sheath thickness, normalizes neurofilament immunoreactivity ${ }^{[19]}$.

A complex dysregulation of the $\mathrm{TH}$ tissue signaling is reported in experimental model of inflammatory/demyelinating diseases. A downregulation of all TRs, including $\mathrm{TR} \alpha$, which is responsible for oligodendroglial lineage induction from neural stem cell as well as TR $\beta$, which is responsible for OPC maturation into oligodendrocytes was found ${ }^{[20]}$. A 30-fold upregulation was found in theT3-inactivating enzyme D3 mRNA, and the ectopic expression of D3 observed also in astrocytes, NG2-IR (nerve glial antigen 2 immunoreactive) OPCs was found. D3 protein strongly upregulates in vitro in astrocytes exposed to proinflammatory cytokines. Alterations in TR and $\mathrm{D}$ expression correlate with the rise of inflammatory cytokines released ${ }^{[21]}$. The overall scenario emerging from in vivo data collected in animal models of inflammatory diseases like that from human patients, namely, D3 overexpression and TR downregulation, supports the hypothesis that inflammation leads to a substantial tissue hypothyroidism. Inflammatory cytokines and HIF (hypoxia inducible factor) are regarded as potential candidates for mediating at least the $\mathrm{D}$ regulation in many experimental models.

\section{Method and Materials}

Patients presenting with neurological manifestations to medical outpatient department evaluated first by detailed general, physical, systemic, and neurological examination. T3 (FT3), T4 (FT4), and (TSH) levels evaluated.

A normal range of TPO-Ab(+) was defined as below $16 \mathrm{IU} / \mathrm{mL}$.

Hypothyroid patients between 35-45 yrs age are subjected to the study after taking written informed consent. They are explained in detail about the test .

50 hypothyroid patients negative for thyroid antibodies as controls.

50 hypothyroid patients positive for thyroid antibodies as cases

After biochemical investigations, electrophysiological studies were done.

Materials :A computer system ; Chart software ; Power Lab (with built-in Bio Amp or Power Lab and Bio Amp front-end); Five-lead Shielded Bio Amp Cable \& snap-connect Shielded Lead Wires; Disposable ECG recording electrodes; Stimulus Bar Electrode ; Electrode gel ; Alcohol swabs; Dry earth.

Watch, jewelry, etc. removed from the wrists. The five-lead Bio Amp cable connected to the bio amp. Dry earth lead connected to earth connection of bio amp cable. Skin cleaned with alcohol, 2 disposable ECG electrodes are attached to the skin overlying the abductor pollicis brevis muscle $2-3 \mathrm{~cm}$ apart after applying small drop of electrode gel. Four shielded lead wires connected to bio amp cable ports for positive and negative, 1 and 2 channels. Stimulus electrode connected to stimulator output of the Power Lab positive to positive and negative 
to negative. Stimulus electrode placed over the median nerve at the wrist along the axis of the arm, with the negative lead towards wrist. The nerve stimulated by a mild and brief single electrical shock from the stimulating electrode and same strength is used for all subjects. The resulting electrical activity is recorded by recording electrodes. Distance between the electrodes and the time taken by electrical impulses to travel between electrodes are used to measure conduction velocities. Amplitudes and latencies are also calculated from the chart. Nerve action potentials were amplified in the conventional way and displayed on power lab monitor. All tests were carried out in a warm room with the subject lying on a couch in relaxed position as records are affected by muscular contraction.

The system is adjusted in such a way that the stimulus takes place after $5 \mathrm{~ms}$ of pressing start button and record is taken for $70 \mathrm{~ms}$. Recording shows two tracings one is stimulus tracing which shows the point of stimulus and with tracing of action potential below it marked by the digital system of the power lab.

\section{Inclusion criteria:}

Subjects attending medical OP with symptoms of peripheral neuropathy without diabetes or any other deficiency disorders like VitB12, alcoholism and thyroid profile showing hypothyroidism and with thyroid antibody test results both positive and negative .

\section{Exclusion criteria:}

Subjects already diagnosed and being treated as diabetes mellitus, alcoholics, people suffering with nutritional deficiencies like Vit B12.

Observational, cross-sectional study was performed in the Department of Physiology in collaboration with Department of Medicine at Shadan Institute of Medical Sciences Hyderabad.

The protocol of the study was approved by the Institutional Ethics Committee.

Informed consent is obtained from all the patients subjected to the test.

\section{Results}

Correlation was observed in antibodies presence and the results. Patients with positive antibodies showed nerve conduction disturbances of increased latency,decreased amplitude and conduction velocity. These findings are in concordance with the well known nerve damage and other laboratory findings in hypothyroidism more commonly in patients diagnosed with autoimmune type of hypothyroidism damage to thyroid gland.

Table 1. Amplitudes, latencies, conduction velocities of motor nerves compared in hypothyroid patients with negative and positive antibodies.

\begin{tabular}{|l|l|l|l|l|}
\hline Parameters & $\begin{array}{l}\text { Hypothyroid with } \\
\text { Negative antibodies } \\
\text { mean (SD) }\end{array}$ & $\begin{array}{l}\text { Hypothyroid with } \\
\text { Positive antibodies } \\
\text { mean (SD) }\end{array}$ & t - test & p - value \\
\hline Amplitude(mV) & & & 7.92 & $<0.001$ \\
\hline Median Nerve & $4.50(0.48)$ & $4.02(0.35)$ & 1.77 & 0.07 \\
\hline Ulnar Nerve & $6.68(0.92)$ & $6.95(1.39)$ & & \\
\hline Conduction Velocities (m/s) & & & 9.03 & $<0.001$ \\
\hline Median Nerve & $59.23(4.67)$ & $53.91(4.45)$ & 2.14 & 0.03 \\
\hline Ulnar Nerve & $54.86(5.21)$ & $53.32(5.90)$ & & $<0.001$ \\
\hline Latency (ms) & & & 5.63 & 0.01 \\
\hline Median Nerve & $2.91(0.63)$ & $3.54(1.05)$ & 2.37 & \\
\hline Ulnar Nerve & $2.76(0.52)$ & $3.03(1.13)$ & & \\
\hline
\end{tabular}


Table 2. Amplitudes, latencies, conduction velocities of sensory nerves compared in hypothyroid patients with negative and positive antibodies.

\begin{tabular}{|l|l|l|l|l|}
\hline Parameters & $\begin{array}{l}\text { Hypothyroid with } \\
\text { negative antibodies } \\
\text { mean(SD) }\end{array}$ & $\begin{array}{l}\text { Hypothyroid with positive } \\
\text { antibodies } \\
\text { mean(SD) }\end{array}$ & t-test & p-value \\
\hline Amplitude (mV) & & & & \\
\hline Median Nerve & $24.72(11.90)$ & $18.35(8.41)$ & 4.78 & $<0.001$ \\
\hline Ulnar Nerve & $20.52(6.30)$ & $18.15(5.71)$ & 3.05 & $<0.01$ \\
\hline Conduction Velocities (m/s) & & & & \\
\hline Median Nerve & $54.47(6.34)$ & $49.25(5.21)$ & 6.96 & $<0.001$ \\
\hline Ulnar Nerve & $50.29(7.45)$ & $44.52(5.00)$ & 7.04 & $<0.001$ \\
\hline Latency (ms) & & & & \\
\hline Median Nerve & $2.29(0.42)$ & $2.70(0.21)$ & 9.56 & $<0.001$ \\
\hline Ulnar Nerve & $3.37(0.51)$ & $4.01(1.23)$ & 5.26 & $<0.001$ \\
\hline
\end{tabular}

\section{Discussion}

Primary hypothyroidism is a chronic and insidious disease caused by failure of thyroid hormone production mostly due to inflammation, thyroidectomy, use of ${ }^{131} \mathrm{I}$, and anti-hyperthyroid drugs. A wide variety of systems may be affected, such as renal, digestive, cardiac, circulatory and nervous. The nervous system may be damaged centrally or peripherally. Dysfunction of the peripheral nerve system (PNS) is not infrequent and should prompt correct diagnosis and treatment of this endocrinopathy when present. Early treatment provides overall clinical amelioration. The so called myxoedematous myopathy represents to $10 \%$ of acquired myopathies. This myopathy, in its full expression, is unusual but by that time, frequent are complaints as weakness, cramps, and stiffness. Slowness of relaxation time of deep reflexes is found in $80 \%$ of the case. Thus, it should be considered that the real incidence of muscle dysfunction may be underestimated. Hypoesthesia, pain and paraesthesias, deep reflexes hyporeflexia, atrophy, and disautonomy may be present in hypothyroidism. The electrophysiological diagnosis of peripheral neuropathy reaches $72 \%$ of cases. The real prevalence of hypothyroidism neuropathy and/or myopathy depends on the diagnostic criteria used, either clinical, electrophysiological, laboratorial, structural or combined. In addition, most available data published are case reports ${ }^{[2]}$.

Peripheral neuropathy has a variety of systemic, metabolic, and toxic causes. The most common treatable causes include diabetes mellitus, hypothyroidism, and nutritional deficiencies. The diagnosis, thus, requires careful clinical assessment, judicious laboratory testing, and electrodiagnostic studies or nerve biopsy Peripheral neuropathy can involve different nerve types including motor, sensory, and autonomic nerves. Peripheral neuropathy can also be categorized by the size of the nerve fibers involved, large or small.

Thyroid hormones exert multiple effects on neural development and function ${ }^{[22]}$. Overt hypothyroidism is associated with significant alterations both in the neuromuscular system and brain functions. The neurological manifestations of clinical hypothyroidism in adults are varied and include peripheral neuropathy, entrapment neuropathy, mental dysfunction, hearing loss, seizures, possibly cerebellar ataxia, and myxedema coma. In some patients with clinical hypothyroidism, peripheral nerves dysfunction may be the main and presenting manifestation. Peripheral neuropathy may be caused by severe, long-term, untreated hypothyroidism. Although the association between hypothyroidism and 
peripheral neuropathy is not fully understood, it is known that hypothyroidism can cause fluid retention resulting in swollen tissues that exert pressure on peripheral nerves.

There are many reports on electroneuromyography (ENMG) changes in hypothyroidism. The objectives of the present study were to relate the signs and symptoms of PNS dysfunction; and compare mean values of nerve conduction studies in patients with neurological abnormalities in hypothyroidism and correlate them with neurological signs and symptoms and thyroid antibody levels; and to compare latency, amplitude and nerve conduction velocity from selected nerves. 100 patients suffering from primary hypothyroidism were submitted to nerve conduction studies. Abnormalities were found in patients positive for thyroid antibodies. Clinical, laboratorial and nerve studies correlation was observed in patients with hypothyroidism and thyroid antibodies. The patients with positive thyroid antibodies showed a significant tendency of nerve conduction slowness as compared without antibodies. The findings are in concordance with the well-known nerve and muscle damage in hypothyroidism.

\section{Conclusion}

Association between Hashimoto's hypothyroidism and peripheral neuropathy is not fully understood and exact role of antithyroid antibodies in the pathogenesis of neuropathy is not precisely clear.

Absence of antecedent infections, fever, diabetes, vitamin deficiencies, hypothyroid profile with antithyroid antibody seropositivity suggests Hashimoto's neuropathy as a possible cause of abnormal nerve conduction studies. Anti-thyroid antibodies might have pathogenic significance and early detection and correction of thyroid deficiency and other treatments for autoimmunity,may help to prevent major complications

Ethical Clearance: Institutional clearance is obtained.

\section{Conflict of Interest: None.}

Source of Funding: None.

\section{References}

1. Calza, L., Fernandez, M., Giuliani, A., D’Intino, G.,
Pirondi, S., Sivilia, S., et al. Thyroid hormone and remyelination in adult central nervous system: A lesson from an inflammatory-demyelinating disease. Brain Research Reviews, 48, 339346,2005 .

2. Little, A. G. A review of the peripheral levels of regulation by thyroid hormone. Journal of Comparative Physiology B, 186, 677-688,2016.

3. Nielsen $\mathrm{CH}$, Brix TH, Leslie RG, et al. A role for autoantibodies in enhancement of pro-inflammatory cytokine responses to a self-antigen, thyroid peroxidase. Clin Immunol ;133:21827,2009.

4. Rc Mcleod DS, Caturegli P, Cooper DS, Motos PG, Hutfless S. Variation in rates of autoimmune thyroid disease by race/ethnicity in US military personnel. JAMA ;311:1563-5,2014. [PMID 24737370].

5. Carlê A, Laurberg P, Knudsen N, et al. Thyroid peroxidase and thyroglobulin auto-antibodies in patients with newly diagnosed overt hypothyroidism. Autoimmunity,39:497503,2006.

6. Lee, J. Y., \& Petratos, S.;Thyroid hormone signaling in oligodendrocytes: From extracellular transport to intracellular signal. Molecular Neurobiology, 53, 6568-6583,2016.

7. PLoS ONE ;10:e0142615. doi:10.1371/journal. pone.0142615,2015. [PMC free article] [PubMed] [CrossRef] [Google Scholar)

8 \&10. Calza, L., Fernandez, M., \& Giardino, L.; Role of the thyroid system in myelination and neural connectivity; Comprehensive Physiology, 5, 1405 1421, 2015.

9. Bernal, J., Guadan o-Ferraz, A., \& Morte, B; Thyroid hormone transporters Functions and clinical implications. Nature Reviews Endocrinology, 11, 406-417,2015.

11. Buser, J. R., Maire, J., Riddle, A., Gong, X., Nguyen, T., Nelson, K., et al. Arrested preoligodendrocyte maturation contributes to myelination failure in premature infants. Annals of Neurology, 71, 93 $-109,2012$.

12\&13. Crawford, A. H., Chambers, C., \& Franklin, R. J. Remyelination: The true regeneration of the central nervous system. Journal of Comparative Pathology, 149, 242-254,2013.

14. Crawford, A. H., Tripathi, R. B., Richardson, W. 
D., \& Franklin, R. J. Developmental origin of oligodendrocyte lineage cells determines response to demyelination and susceptibility to age-associated functional decline. Cell Reports, 761-773. http:// dx.doi. org/10.1016/j.celrep.2016.03.069, 2016.

15. Franklin, R. J., \& Ffrench-Constant, C. ; Remyelination in the CNS: From biology to therapy. Nature Reviews Neuroscience, 9, 839-855, 2008.

16\&17. D’Intino, G., Lorenzini, L., Fernandez, M., Taglioni, A., Perretta, G., Del Vecchio, G., et al.; T3 administration ameliorates the demyelination/ remyelination ratio by correcting tissue hypothyroidism. Journal of Neuroendocrinology, 23, 778-790,2011.
18. Zhang, M., Ma, Z., Qin, H., Yao, Z. Thyroid hormone potentially benefits via facilitating remyelination. Molecular Neurobiology, 53, 4406-4416,2015.

19,20\&21. Fernandez, M., Giuliani, A., Pirondi, S., D’Intino, G., Giardino, L., Aloe, L., et al., Thyroid hormone administration enhances remyelination in chronic demyelinating inflammatory disease. Proceedings of the National Academy of Sciences of the United States of America, 101, 1636316368,2004 .

22. Boyages SC. The neuromuscular system and brain in hypothyroidism. In: Braverman LE, Utiger RD, editors.Werner and Ingbar's the Thyroid: A Fundamental andClinicalText. $8^{\text {th }}$ ed. Philadelphia: Lippincott Williams \& Wilkins; p. 803-10,2000. 